Article

\title{
Large-Signal Stability Analysis of the Undersea Direct Current Power System for Scientific Cabled Seafloor Observatories
}

\author{
Yamei Jiang ${ }^{1,2}$ and Feng Lyu 1,2,3,*(D) \\ 1 State Key Laboratory of Marine Geology, Tongji University, Shanghai 200092, China \\ 2 School of Ocean and Earth Science, Tongji University, Shanghai 200092, China \\ 3 Center for Marine Science and Technology, Tongji University, Shanghai 200092, China \\ * Correspondence: LF@tongji.edu.cn
}

Received: 15 June 2019; Accepted: 30 July 2019; Published: 2 August 2019

check for updates

Featured Application: This technology provides a feasible approach for analyzing and improving the large-signal stability of the undersea direct current power system for scientific cabled seafloor observatories. It is of great significance for the design, construction, operation and maintenance of the cabled seafloor observatories.

\begin{abstract}
A large number of power electronic converters and long-distance submarine cables are an important part of the undersea direct current (DC) power system of the scientific cabled seafloor observatories (CSOs). However, the constant power load (CPL) characteristics of the converters and the distributed parameter characteristics of long-distance submarine cables greatly affect the stability of the CSO DC power system. This paper analyzes the large-signal stability of the CSO DC power system, and the equivalent circuits of long-distance submarine cables are established by theoretical analysis and computer simulation. A simplified computer simulation model and an equivalent experimental prototype model of a single-node CSO DC power system was built in the laboratory to study this issue. The mixed potential function method is used to analyze the large-signal stability of the CSO DC power system, and the large-signal stability criterion is obtained theoretically. The validity of the large-signal stability criterion is proved by simulations and experiments. The conclusion is that reducing the inductance of the submarine cable, increasing the capacitance of the submarine cable, increasing the output voltage of the shore station power feeding equipment (PFE) or reducing the power consumption of the undersea station, are beneficial to improve the large-signal stability of the CSO DC power system.
\end{abstract}

Keywords: cabled seafloor observatories; direct current power systems; large-signal stability; mixed potential function

\section{Introduction}

Since ancient times, mankind has never given up on the exploration of the ocean, and the tools for observing the ocean have been constantly innovating, such as the scientific cabled seafloor observatories (CSOs). It is an emerging means of ocean observation that collects continuous real-time data over long periods by laying a regional observing system on the ocean floor.

Since 2006, more and more scientific CSOs have been established around the world for oceanography research, e.g., MARS (Monetary Accelerated Research System) [1], OOI (Ocean Observatory Initiative) [2], ALOHA cabled observatory [3], VENUS (Victoria Experimental Network Under the Sea) [4], NEPTUNE (North-East Pacific Time-series Underwater Networked Experiment) [5], DONET (Dense Oceanfloor Network System for Earthquakes and Tsunamis) [6], S-net (Seafloor 
observation network for earthquakes and tsunamis along the Japan Trench) [7], ESONET (European Seas Observatory Network) [8], Xiaoqushan seafloor observatory [9] and MACHO (Marine Cable Hosted Observatory) [10]. Moreover, the East China Sea (ECS) and the South China Sea (SCS) experimental observatories were built in 2015 and 2016, respectively. In 2017, the Chinese national scientific seafloor observatory (CNSSO) was launched to realize the long-term observation of the complex processes operating within the oceans.

In typical scientific CSOs, the shore station converts the alternating current (AC) power from the terrestrial electrical grid into the direct current (DC) power, rated up to $-10 \mathrm{kV}$ and delivers it to the undersea stations through backbone cables, branching units (BUs) and spur cables, as shown in Figure 1 [11]. A DC/DC converter is installed in each undersea station, converting $-10 \mathrm{kV}$ DC power from the spur cable into $375 \mathrm{~V}$ DC power for scientific instrument interface modules (SIIMs). The SIIMs convert $375 \mathrm{~V}$ DC power into DC power under $48 \mathrm{~V}$ required by science instruments (SIs). In this way, the long-distance electrical power transmission and conversion for undersea distributed science payloads are completed.

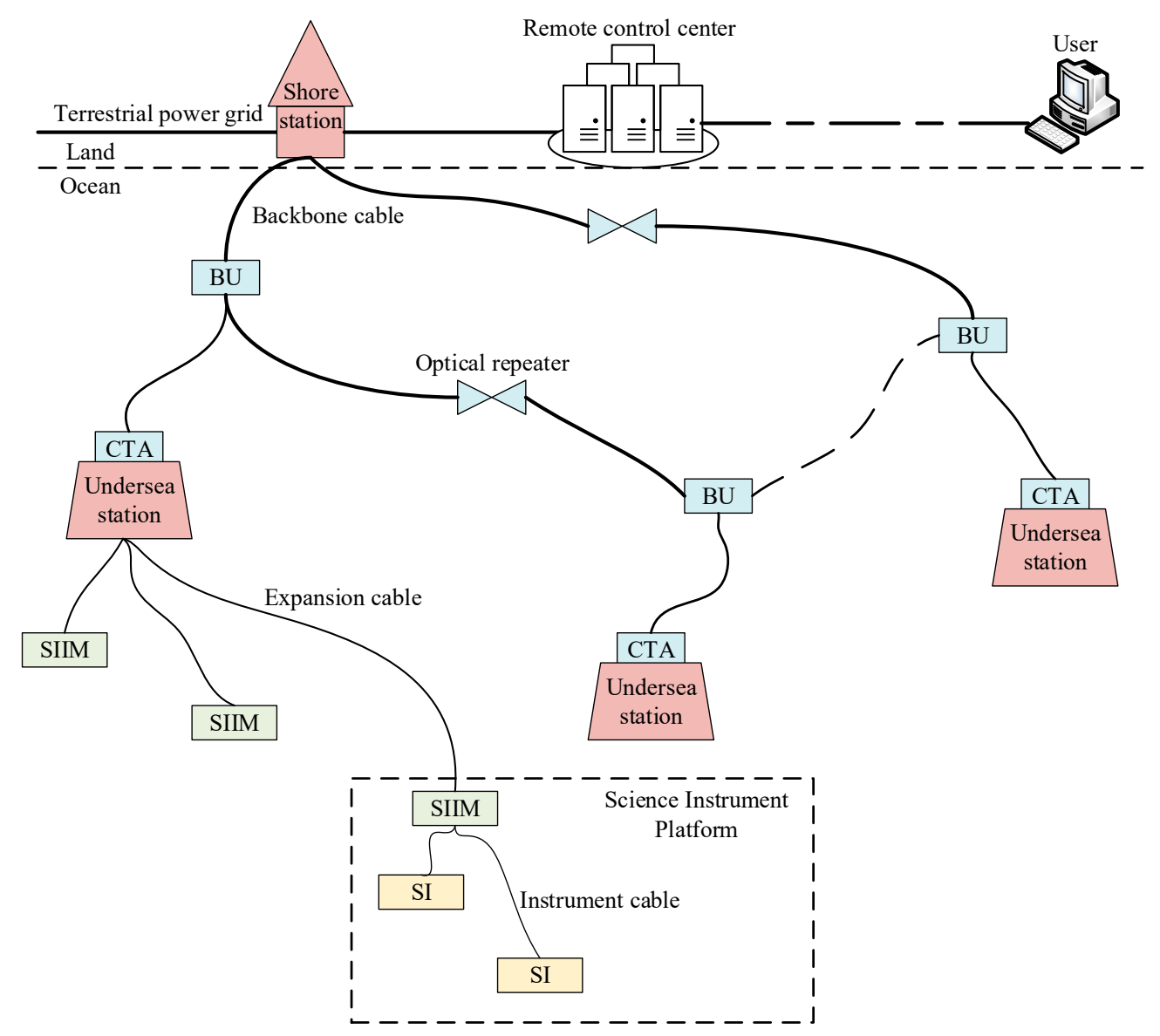

Figure 1. A typical system diagram of scientific cabled seafloor observatories (CSOs).

An undersea DC power system of scientific CSO has a large-scale DC distributed power system (DPS) that contains many cascaded power electronic converters, connected by hundreds to thousands of kilometers of submarine cables [12]. Each point of load (POL) converter, installed in undersea stations, acts as a constant power load (CPL), and the distributed parameter characteristics of long-distance submarine cables greatly affect the stability of the undersea DC power system [12-15]. In the event of instability, the undersea DC power system will oscillate, or even collapse, especially under large disturbance conditions. Because of the harsh subsea environment, CSOs have low accessibility and are 
very expensive to construct and repair, time-consuming and labor-intensive. Therefore, the stability of the CSO undersea DC power system must be analyzed during design and operation.

At present, there is less stability analysis of the undersea DC power system of CSOs worldwide. In 2006, Dr. Lu analyzed the stability of the NEPTUNE observatory power system, but it was lacking in simulation and experimental verification [12]. At present, the stability has been studied in small-scale DC DPSs, e.g., space stations, aircraft, ships and electric vehicles [15-19]. Compared to these DC DPSs, the undersea DC power system of scientific CSOs are more complicated, mainly because of the long-distance submarine cables.

This paper focuses on the large-signal stability of the CSO undersea DC power system, which is a high-order nonlinear system. Large-signal stability means that the power system can go back to its original equilibrium state or transition to a new equilibrium state, after encountering a large disturbance during normal operating conditions [20]. For second or lower-order nonlinear systems, large-signal stability analysis can use the phase plane method [19]. For higher-order nonlinear systems, large-signal stability analysis can be performed by the Lyapunov direct method [20], the key of which is to construct a suitable Lyapunov function. Various approaches have been proposed to produce Lyapunov functions, e.g., the Brayton-Moser mixed potential function method [21,22], the Takagi-Sugeno multi-modeling method [23], genetic algorithm methods [24] and the reverse trajectory tracking method [25]. The literature [26] compares the above several methods. The Takagi-Sugeno multi-modeling method uses multiple linear models to fit nonlinear models, and is not suitable for higher-order nonlinear systems. The Brayton-Moser mixed potential function method needs to find the voltage and current potential functions, which is conservative. The genetic algorithm method requires an artificial neural network toolbox and selects appropriate parameters. The reverse trajectory tracking method can only be represented by an image, and the analytical expression cannot be obtained. In this paper, the mixed potential function method is applied to consider the effect of long-distance submarine cables on the power system large-signal stability, and the equivalent circuits of a long-distance submarine cable are built. Based on this, the equivalent topology model of a single-node CSO undersea DC power system is established. Considering the characteristics of long-distance submarine cables, the large-signal stability of the single-node CSO undersea DC power system is analyzed by the mixed potential function method, and the large-signal stability criterion is obtained. The large-signal stability criterion is verified by computer simulations and experiments, and the factors affecting the large-signal stability of the CSO undersea DC power system are analyzed by simulations and experiments, which is of great significance to the design, operation and future development of the CSO undersea DC power system.

The remainder of the paper is divided as follows. Section 2 introduces the equivalent circuits of a long-distance submarine cable. Section 3 analyses the steady-state equilibrium point and the large-signal stability criterion of the CSO undersea DC power system. Section 4 simulates and experiments on the large-signal stability of CSO undersea DC power systems. Section 5 summarizes the conclusion and anticipates further research on this topic.

\section{The Equivalent Circuits of a Long-distance Submarine Cable}

In scientific CSOs, submarine electro-optic cables are used to transmit electrical power to the seafloor, and mainly consist of the insulating sheath, the composite conductor, the steel wires strand, the steel tube, the thixotropic jelly and optical fibers [12].

According to the electrical conductivity and radius of different materials in submarine cables, the resistance, inductance and capacitance of per unit length submarine cable can be obtained by theoretical calculation, shown in Table 1 for a typical submarine cable [12,27]. In Table $1, R_{1}, L_{1}$ and $C_{1}$ represent the resistance, the inductance and the capacitance of $1 \mathrm{~km}$ submarine cable, respectively. 
Table 1. The parameters of $1 \mathrm{~km}$ typical submarine cable.

\begin{tabular}{cccc}
\hline Parameters & $\mathbf{R}_{\mathbf{1}}$ & $\mathbf{L}_{\mathbf{1}}$ & $\mathbf{C}_{\mathbf{1}}$ \\
\hline Values & $1 \Omega$ & $0.4 \mathrm{mH}$ & $0.2 \mu \mathrm{F}$ \\
\hline
\end{tabular}

The lumped parameter equivalent circuits of long-distance submarine cables are the approximation of the actual distributed characteristics within a certain range. In our studies, multiple $\pi$-type lumped parameter models are cascaded to simulate a long-distance submarine cable. The length of each cable segment represented by a $\pi$-type lumped parameter model, as shown in Figure 2, will affect the accuracy of the equivalent circuits of a submarine cable.

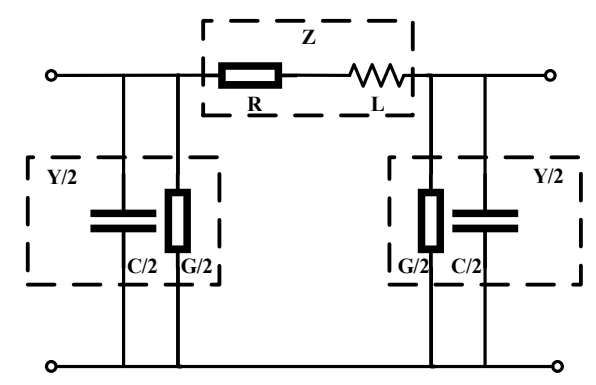

Figure 2. The $\pi$-type lumped parameter model of a submarine cable segment.

The two-port network of a submarine cable can be expressed as

$$
\left\{\begin{array}{c}
Z=Z_{C} \sinh (\gamma l) \\
Y=\frac{1}{Z_{C}} \frac{2(\cosh \gamma l-1)}{\sinh \gamma l} \\
\omega=2 \pi f \\
z=R+j \omega L \\
y=G+j \omega C \\
Z_{C}=\sqrt{\frac{z}{y}} \\
\gamma=\sqrt{y z}
\end{array}\right.
$$

where $f$ is the characteristic frequency of the undersea DC power system, $f=1 \mathrm{kHz}$ in our study. $\omega$ is the angular velocity of the undersea DC power system. $l$ is the overall length of the submarine cable. $R, L, G$ and $C$ are corresponding to the equivalent resistance, inductance, conductance and capacitance of the submarine cable, respectively. The insulation resistance of submarine cables is very high, so that the conductance between the copper conductor and the seawater can be negligible, that is, $G=0 . z$ and $y$ are the impedance and admittance of per unit length submarine cable, respectively. $Z_{C}$ is the wave impedance. $\gamma$ is the propagation coefficient.

When $\gamma l<<1, Z$ and $Y$ can be expressed approximately as

$$
\begin{aligned}
& Z \approx z l \\
& Y \approx y l .
\end{aligned}
$$

In order to study the influence of the cable segment length on the model accuracy, the two-port network model of the submarine cable is regarded to be accurate as a reference, and the $\pi$-type lumped parameter models of different cable segment lengths are taken as approximations. The comparison of the two-port network and lumped parameter model under different cable segment lengths are 
shown in Table 2. When multiple $\pi$-type lumped parameter models are cascaded to simulate a long-distance submarine cable, the longer the cable segment represented by each $\pi$-type equivalent circuit, the greater the model parameter error is between the actual submarine cable characteristics and the equivalent circuits.

Table 2. The comparison of the two-port network and the lumped parameter model under different cable segment lengths.

\begin{tabular}{ccccc}
\hline$f=\mathbf{1 ~ k H z}$ & $\begin{array}{c}Z \text { (Two-Port } \\
\text { Network) }\end{array}$ & $\begin{array}{c}\boldsymbol{Z} \text { (Lumped } \\
\text { Model) }\end{array}$ & $\boldsymbol{Y}$ (Two-Port Network) & $\begin{array}{c}\boldsymbol{Y} \text { (Lumped } \\
\text { Model) }\end{array}$ \\
\hline$l=1 \mathrm{~km}$ & $1.00+2.51 \mathrm{i}$ & $1.00+2.51 \mathrm{i}$ & $1.32 \times 10^{-7}+1.26 \mathrm{i} \times 10^{-3}$ & $1.26 \mathrm{i} \times 10^{-3}$ \\
$l=2 \mathrm{~km}$ & $1.99+5.02 \mathrm{i}$ & $2.00+5.03 \mathrm{i}$ & $1.06 \times 10^{-6}+2.52 \mathrm{i} \times 10^{-3}$ & $2.51 \mathrm{i} \times 10^{-3}$ \\
$l=3 \mathrm{~km}$ & $2.97+7.51 \mathrm{i}$ & $3.00+7.54 \mathrm{i}$ & $3.57 \times 10^{-6}+3.78 \mathrm{i} \times 10^{-3}$ & $3.77 \mathrm{i} \times 10^{-3}$ \\
$l=4 \mathrm{~km}$ & $3.93+9.98 \mathrm{i}$ & $4.00+10.05 \mathrm{i}$ & $8.51 \times 10^{-6}+5.05 \mathrm{i} \times 10^{-3}$ & $5.03 \mathrm{i} \times 10^{-3}$ \\
$l=5 \mathrm{~km}$ & $4.87+12.43 \mathrm{i}$ & $5.00+12.57 \mathrm{i}$ & $1.67 \times 10^{-5}+6.33 \mathrm{i} \times 10^{-3}$ & $6.28 \mathrm{i} \times 10^{-3}$ \\
$l=6 \mathrm{~km}$ & $5.77+14.84 \mathrm{i}$ & $6.00+15.08 \mathrm{i}$ & $2.91 \times 10^{-5}+7.61 \mathrm{i} \times 10^{-3}$ & $7.54 \mathrm{i} \times 10^{-3}$ \\
$l=7 \mathrm{~km}$ & $6.64+17.21 \mathrm{i}$ & $7.00+17.59 \mathrm{i}$ & $4.66 \times 10^{-5}+8.91 \mathrm{i} \times 10^{-3}$ & $8.80 \mathrm{i} \times 10^{-3}$ \\
$l=8 \mathrm{~km}$ & $7.47+19.54 \mathrm{i}$ & $8.00+20.11 \mathrm{i}$ & $7.02 \times 10^{-5}+1.00 \mathrm{i} \times 10^{-2}$ & $1.00 \mathrm{i} \times 10^{-2}$ \\
$l=9 \mathrm{~km}$ & $8.25+21.81 \mathrm{i}$ & $9.00+22.62 \mathrm{i}$ & $1.01 \times 10^{-4}+1.10 \mathrm{i} \times 10^{-2}$ & $1.10 \mathrm{i} \times 10^{-2}$ \\
$l=10 \mathrm{~km}$ & $8.97+24.03 \mathrm{i}$ & $10.00+25.13 \mathrm{i}$ & $1.40 \times 10^{-4}+1.30 \mathrm{i} \times 10^{-2}$ & $1.30 \mathrm{i} \times 10^{-2}$ \\
\hline
\end{tabular}

Computer simulation is performed by using MATLAB. The simulation file is shown in Figure 3. The output voltage of the shore station power feeding equipment (PFE) is $10 \mathrm{kV}$, and the rising time of the output voltage is $1 \mathrm{~ms}$. The length of the submarine cable is $200 \mathrm{~km}$, and the load is $200 \Omega$.

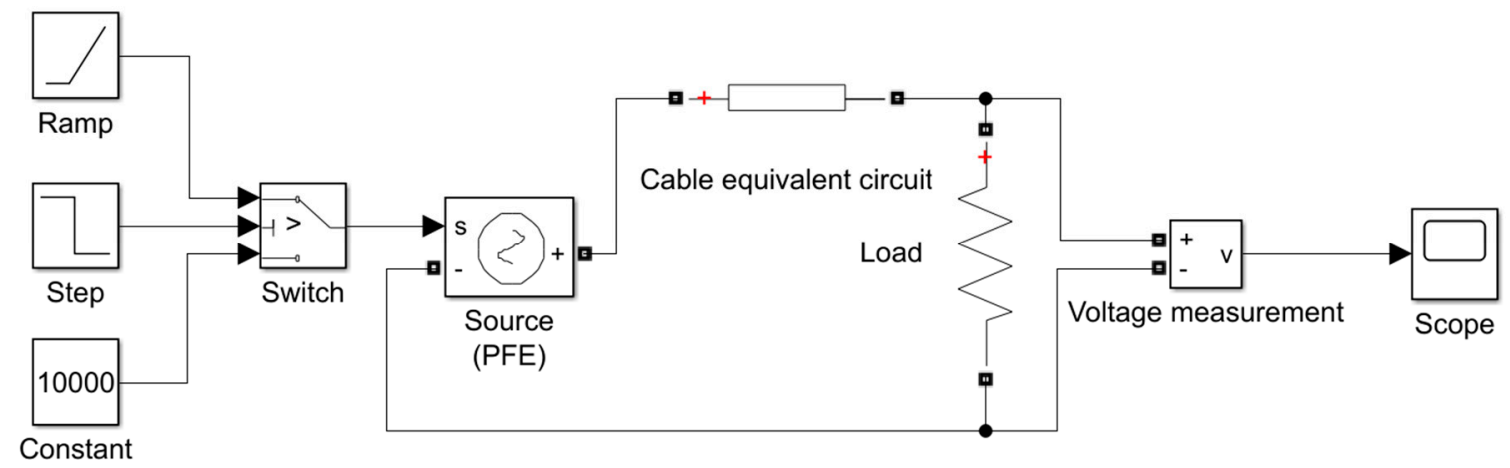

Figure 3. The simulation file for comparing different submarine cable models.

The simulation results are shown in Figure 4. The red line indicates the distributed parameter model of the submarine cable, closest to the actual submarine cable characteristics. The blue, orange, purple and green lines indicate the lumped parameter models of the submarine cable, formed by cascaded 200, 100, 40 and $20 \pi$-type lumped parameter equivalent circuits, respectively. Each $\pi$-type lumped parameter model represents $1 \mathrm{~km}, 2 \mathrm{~km}, 5 \mathrm{~km}$ or $10 \mathrm{~km}$ submarine cable segment.

Taking the red line as a reference, it can be seen that the differences between the blue, orange and red lines are small, and the errors are less than $0.5 \%$. The difference between the purple and red lines is larger, and the error is about $1.5 \%$. The difference between the green and red lines is even larger, and the error is about $6 \%$. It shows that the shorter the submarine cable segment represented by each $\pi$-type lumped parameter equivalent circuit, the smaller the difference between the lumped and distributed parameter models, which is consistent with the results of the above theoretical analysis. 


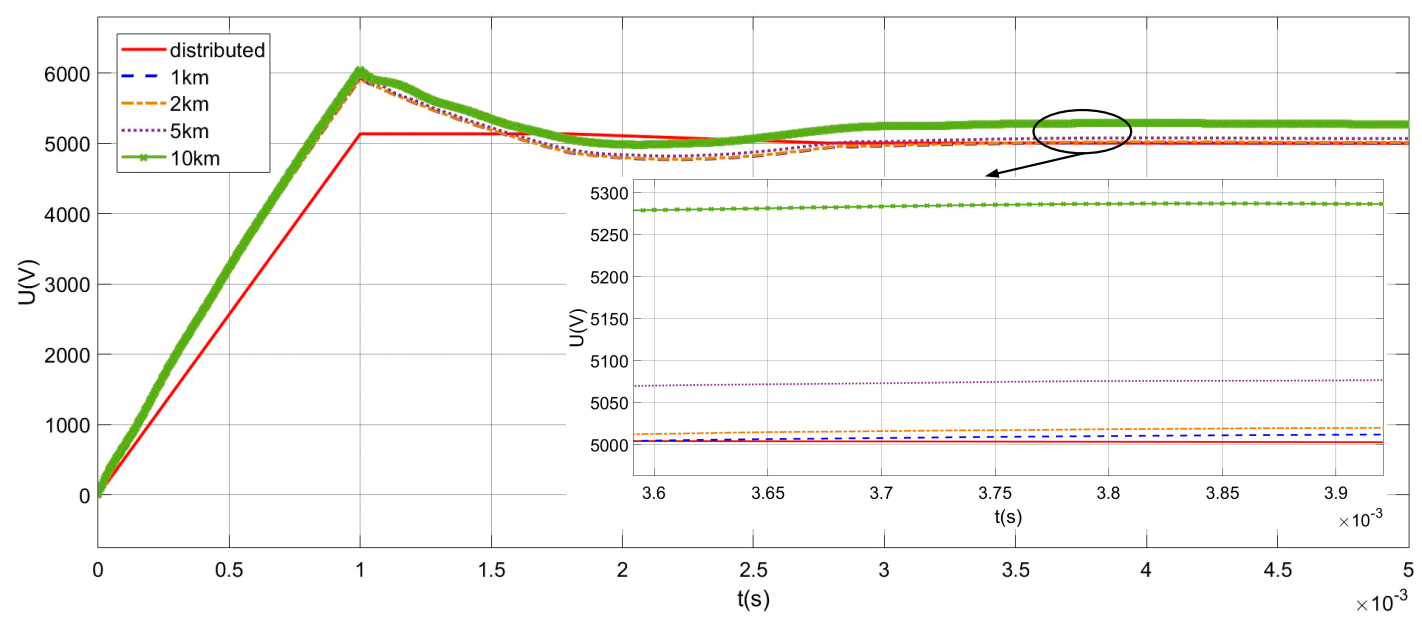

Figure 4. The simulation results of different submarine cable models.

\section{Theoretical Analysis of Undersea DC Power System Stability}

\subsection{Steady-State Equilibrium Point}

In order to analyze the steady-state equilibrium point of a multi-node CSO undersea DC power system, it is necessary to establish a simplified system model, that is, one long-distance submarine cable, one shore station PFE and one undersea station, as shown in Figure 5. The shore station PFE is modeled by an ideal voltage source. The submarine cable is modeled by cascaded lumped parameter circuits. The undersea station is modeled by a CPL.

In Figure $5, U_{S}$ represents the output voltage of the shore station PFE. $R_{0}, L_{0}$ and $C_{0}$ represent the resistance, inductance and capacitance of a cable segment, respectively. $P_{L}, U_{L}$ and $I_{L}$ represent the power consumption, input voltage and input current of the undersea station, respectively, and $n$ represents the number of submarine cable segments.

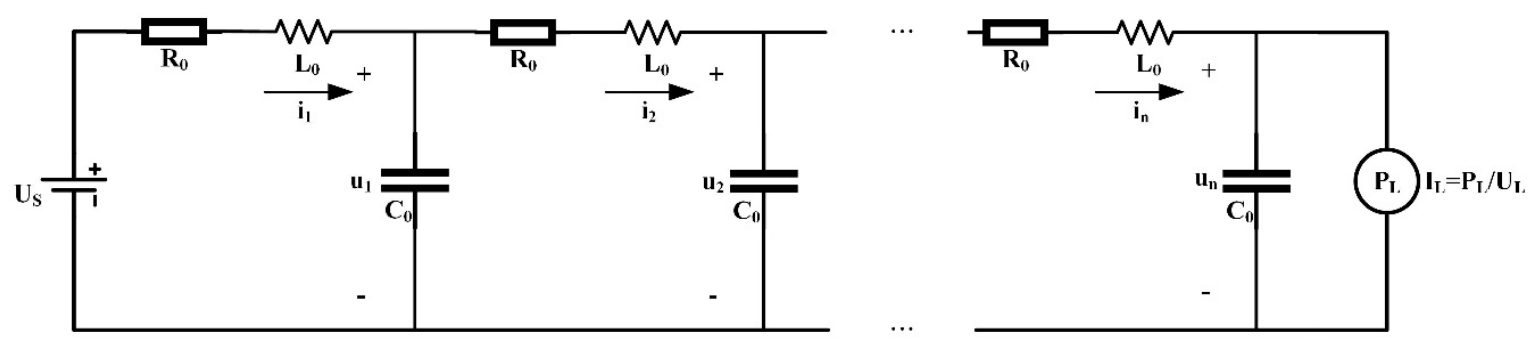

Figure 5. A simplified model of a single-node CSO undersea DC power system.

The steady-state equation of the simplified system model is as follows:

$$
\left\{\begin{array}{l}
U_{L}=U_{S}-n R_{0} I_{L} \\
I_{L}=\frac{P_{L}}{U_{L}}
\end{array}\right.
$$

The equilibrium point of the simplified system model is:

$$
\left\{\begin{array}{l}
U_{L}=\frac{U_{S} \pm \sqrt{U_{S}^{2}-4 n R_{0} P_{L}}}{2} \\
I_{L}=\frac{P_{L}}{U_{L}}
\end{array}\right.
$$

In order to avoid complex roots, it must satisfy the following inequality:

$$
R_{0}<\frac{U_{S}^{2}}{4 n P_{L}}
$$


which is the steady-state equilibrium point criterion for the simplified model of a single-node undersea DC power system.

A medium-voltage experimental prototype of a single-node CSO undersea DC power system was built in the laboratory, as shown in Figure 6. The parameters of each component in the medium-voltage experimental prototype are shown in Table 3.

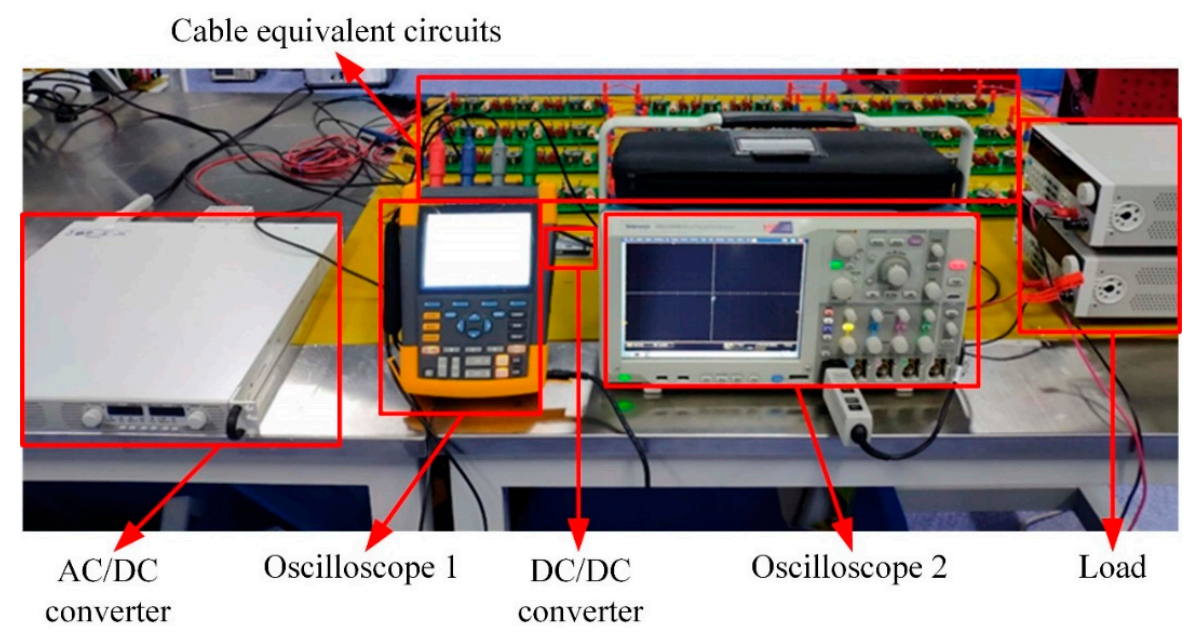

Figure 6. The medium-voltage experimental prototype of a single-node CSO undersea DC power system.

Substituting the values of Table 3 into the inequality Equation (11), it can be obtained:

$$
\left\{\begin{array}{l}
R_{0}=10 \\
\frac{U_{S}^{2}}{4 n P_{L}}=13.23
\end{array}\right.
$$

Table 3. The parameters of each part in the medium-voltage experimental prototype.

\begin{tabular}{ccccccc}
\hline Parameters & $\boldsymbol{U}_{\boldsymbol{S}}$ & $\boldsymbol{n}$ & $\boldsymbol{R}_{\mathbf{0}}$ & $\boldsymbol{L}_{\mathbf{0}}$ & $\boldsymbol{C}_{\mathbf{0}}$ & $\boldsymbol{P}_{\boldsymbol{L}}$ \\
\hline Values & $400 \mathrm{~V}$ & 36 & $10 \Omega$ & $4 \mathrm{mH}$ & $2 \mu \mathrm{F}$ & $84 \mathrm{~W}$ \\
\hline
\end{tabular}

So, the steady-state equilibrium point criterion is satisfied, which indicates that the medium-voltage experimental prototype has steady-state equilibrium points and can operate normally without disturbances.

\subsection{Mixed Potential Function Method}

Brayton and Moser proposed three mixed potential function theorems for analyzing nonlinear RLC networks $[21,22,28]$. Based on the state equation of the power system, a mixed potential function should be constructed to judge the system stability under large disturbances. The CSO undersea DC power system is a topological complete RLC circuit, and its dynamic performance can be described by a mixed potential function.

The mixed potential function $P(i, u)$ can be uniformly expressed as

$$
P(i, u)=-A(i)+B(u)+D(i, u)
$$

where, $-A(i)$ represents the current potential function of some non-energy storage elements in the circuit, $B(u)$ represents the voltage potential function of non-energy storage elements in the circuit and $D(i, u)$ represents the energy of capacitors and part of non-energy storage elements in the circuit $[29,30]$. 
In this paper, the mixed potential function third theorem is used to analyze the large-signal stability of the CSO undersea DC power system with long-distance submarine cables [21]. For the convenience of application, the third theorem is introduced as follows:

$$
\begin{gathered}
P_{u}=\frac{\partial P(i, u)}{\partial u} \\
P_{i}=\frac{\partial P(i, u)}{\partial i} \\
A_{i i}(i)=\frac{\partial^{2} A(i)}{\partial i^{2}} \\
B_{u u}(u)=\frac{\partial^{2} B(u)}{\partial u^{2}}
\end{gathered}
$$

$L_{n}$ and $C_{n}$ are diagonal arrays of branch inductance and branch capacitance, respectively. So,

$$
\begin{aligned}
& \mu_{1}=\min \left\{\lambda\left(L_{n}{ }^{-\frac{1}{2}} A_{i i}(i) L_{n}{ }^{-\frac{1}{2}}\right)\right\} \\
& \mu_{2}=\min \left\{\lambda\left(C_{n}^{-\frac{1}{2}} B_{u u}(u) C_{n}^{-\frac{1}{2}}\right)\right\}
\end{aligned}
$$

where $\lambda(M)$ represents the eigenvalue of the matrix $\mathrm{M}$. In case

$$
\mu_{1}+\mu_{2}>0
$$

and when $|i|+|u| \rightarrow \infty$,

$$
P^{*}(i, u)=\frac{\mu_{1}-\mu_{2}}{2} P(i, u)+\frac{1}{2}\left(P_{i}, L_{n}{ }^{-1} P_{i}\right)+\frac{1}{2}\left(P_{u}, C_{n}{ }^{-1} P_{u}\right) \rightarrow \infty .
$$

When $t \rightarrow \infty$, the system is gradually stable over a wide range. It should be pointed out that this conclusion is only a sufficient condition, not a necessary condition.

For the circuit shown in Figure 5, assuming that $u_{0}=U_{S}$, the following mixed potential function can be derived:

$$
P(i, u)=\sum_{k=1}^{n}\left[-\frac{1}{2} R_{0} i_{k}^{2}+i_{k}\left(u_{k-1}-u_{k}\right)\right]+\int_{0}^{u_{n}} \frac{P_{L}}{u} d u .
$$

It satisfies the following equation:

$$
\left\{\begin{array}{l}
L_{0} \frac{d i_{k}}{d t}=\frac{\partial P}{\partial i_{k}}, k=1,2, \cdots, n \\
-C_{0} \frac{d u_{k}}{d t}=\frac{\partial P}{\partial u_{k}}, k=1,2, \cdots, n
\end{array} .\right.
$$

From Equation (22), it can be derived that:

$$
\begin{gathered}
A_{i i}(i)=\left[\begin{array}{ccccc}
R_{0} & 0 & \cdots & 0 & 0 \\
0 & R_{0} & \cdots & 0 & 0 \\
\vdots & \vdots & \ddots & \vdots & \vdots \\
0 & 0 & \cdots & R_{0} & 0 \\
0 & 0 & \cdots & 0 & R_{0}
\end{array}\right] \\
B_{u u}(u)=-\frac{P_{L}}{u_{n}^{2}}=-\frac{1}{R_{L}}
\end{gathered}
$$


where $-R_{L}$ is the dynamic negative impedance of the CPL, and it can be calculated that

$$
\begin{gathered}
\mu_{1}=\frac{R_{0}}{L_{0}}, \\
\mu_{2}=-\frac{1}{C_{0} R_{L}} .
\end{gathered}
$$

For the system operating at the steady-state equilibrium point, in order to restore stable operation under large disturbances, $\mu_{1}$ and $\mu_{2}$ must satisfy Equations (20) and (21), i.e.,

$$
\frac{C_{0}}{L_{0}}>\frac{1}{R_{0} R_{L}} .
$$

Combining the large-signal stability criterion Equation (28) with the steady-state equilibrium point criterion Equation (11), we can get:

$$
\frac{L_{0}}{C_{0} R_{L}}<R_{0}<\frac{U_{S}{ }^{2}}{4 n P_{L}} .
$$

From the inequality Equation (29), the influence of the submarine cable parameters, the output voltage of the shore station PFE and the load power consumption on the large-signal stability can be qualitatively seen that the large-signal stability of the CSO undersea DC power system is positively correlated with the output voltage of the shore station PFE and the capacitance of the submarine cable and negatively correlated with the power consumption of the undersea station and the inductance of the submarine cable.

\section{Simulation and Experiment}

\subsection{Large-Signal Stability Simulations of a Single-Node CSO Undersea DC Power System}

In this paper, the large-signal stability of a single-node CSO undersea DC power system is simulated by using Saber. The simulation file is shown in Figure 7. The shore station PFE is modeled by an ideal voltage source. The submarine cable is modeled by cascaded lumped parameter equivalent circuits. The undersea station is modeled by a CPL. The total length of the submarine cable is assumed to be $360 \mathrm{~km}$. Every cable segment of $10 \mathrm{~km}$ is represented by a $\pi$-type lumped parameter equivalent circuit.

In order to study the effects of the output voltage of the shore station PFE, the power consumption of the undersea station and the submarine cable parameters on the large-signal stability of a single-node CSO undersea DC power system, five simulation sets are needed. The parameter sets for Saber simulation are shown in Table $4 . U_{S}$ represents the output voltage of the source, i.e., the shore station PFE. $R_{0}, L_{0}$, and $C_{0}$ represent the resistance, inductance and capacitance of a cable segment, respectively. $P_{\mathrm{L}}$ represents the power consumption of the CPL, i.e., the undersea station with external science payloads.

Table 4. Five Saber parameter sets for large-signal stability simulation of a single-node CSO undersea DC power system.

\begin{tabular}{cccccc}
\hline No. & $\boldsymbol{U}_{\boldsymbol{S}} / \mathbf{V}$ & $\boldsymbol{P}_{\boldsymbol{L}} / \mathbf{W}$ & $\boldsymbol{R}_{\mathbf{0}} / \boldsymbol{\Omega}$ & $\boldsymbol{L}_{\mathbf{0}} / \mathbf{m H}$ & $\boldsymbol{C}_{\mathbf{0}} / \boldsymbol{\mu} \mathbf{F}$ \\
\hline$\# 1$ & 400 & 84 & 10 & 4 & 2 \\
$\# 2$ & 400 & 84 & 10 & 400 & 2 \\
$\# 3$ & 400 & 84 & 10 & 4 & 0.0164 \\
$\# 4$ & 350 & 84 & 10 & 4 & 2 \\
$\# 5$ & 400 & 112 & 10 & 4 & 2 \\
\hline
\end{tabular}




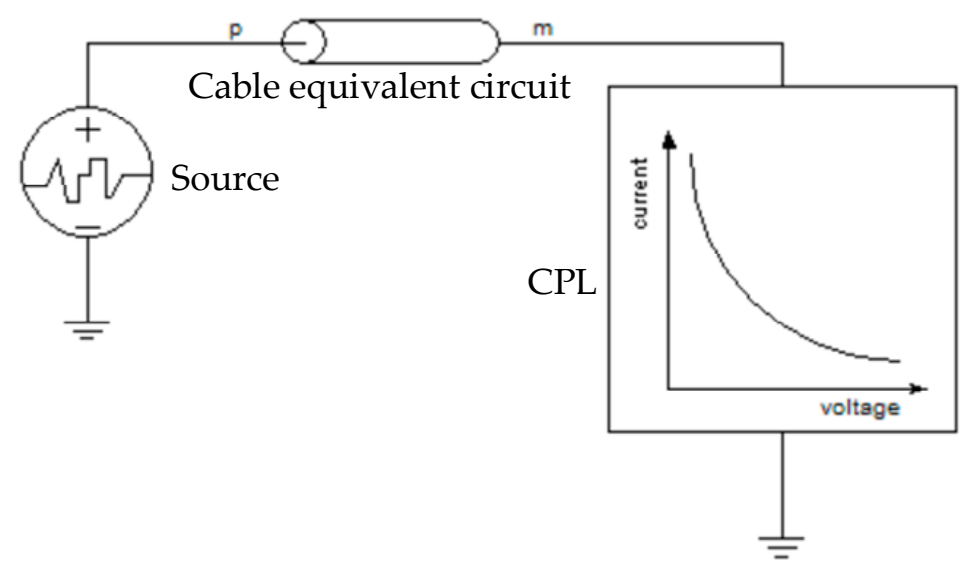

Figure 7. The Saber file for the large-signal stability simulation of a single-node CSO undersea DC power system.

Initially, $U_{S}$ in the system is $0 \mathrm{~V}$. At $0.1 \mathrm{~s}$, according to experimental needs, the voltage rises from $0 \mathrm{~V}$ to $400 \mathrm{~V}$ or $350 \mathrm{~V}$, and the voltage and current transient processes are generated. The simulation results are shown in Figure 8. The blue line indicates the output voltage of the shore station PFE. The green line indicates the voltage in the middle section of the submarine cable. The orange line indicates the input voltage of the undersea station. The red line indicates the output current of the shore station PFE.

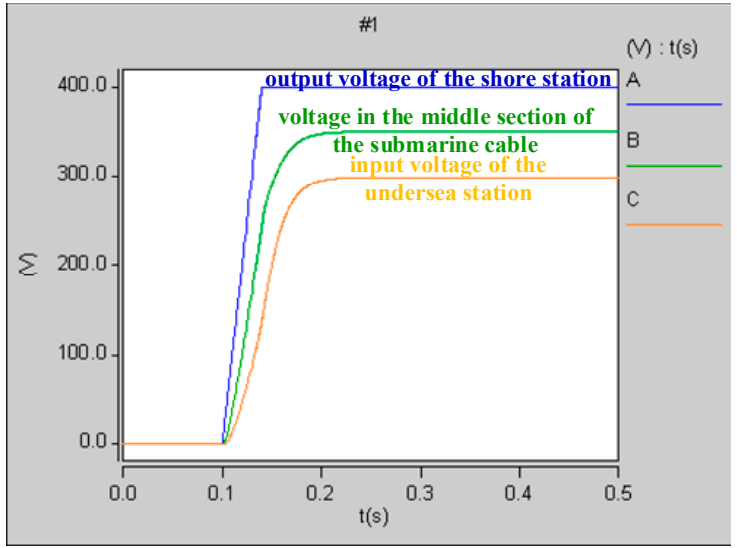

(a)

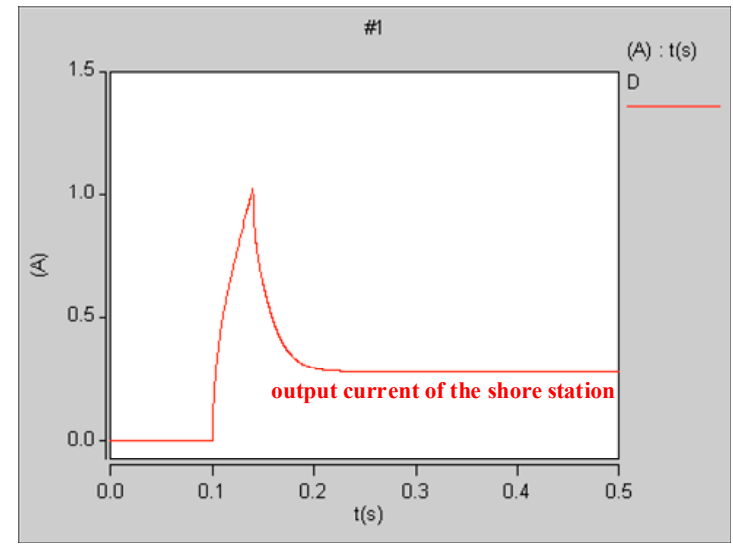

(b)

Figure 8. Cont. 


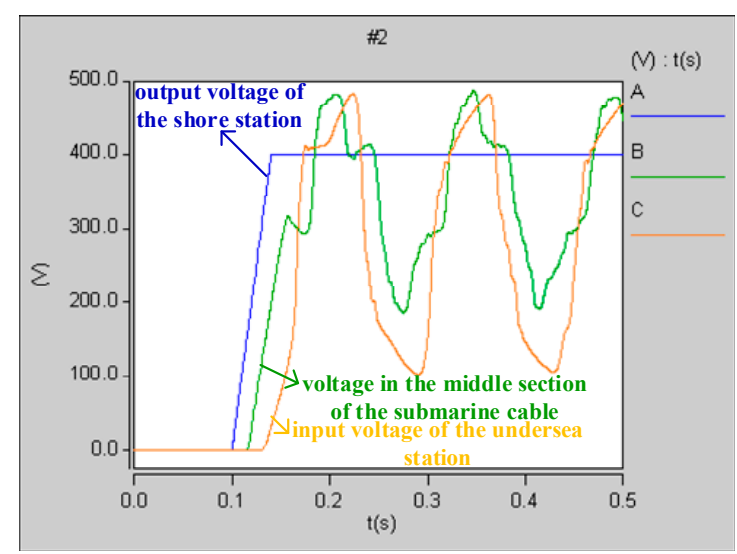

(c)

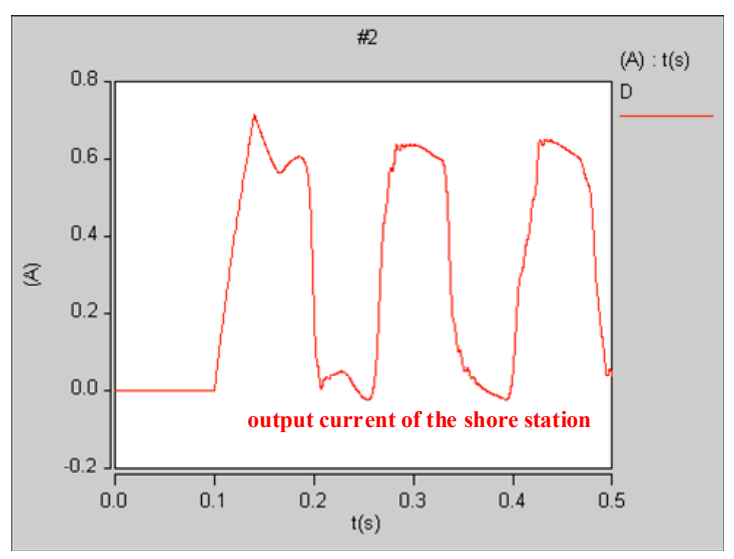

(d)

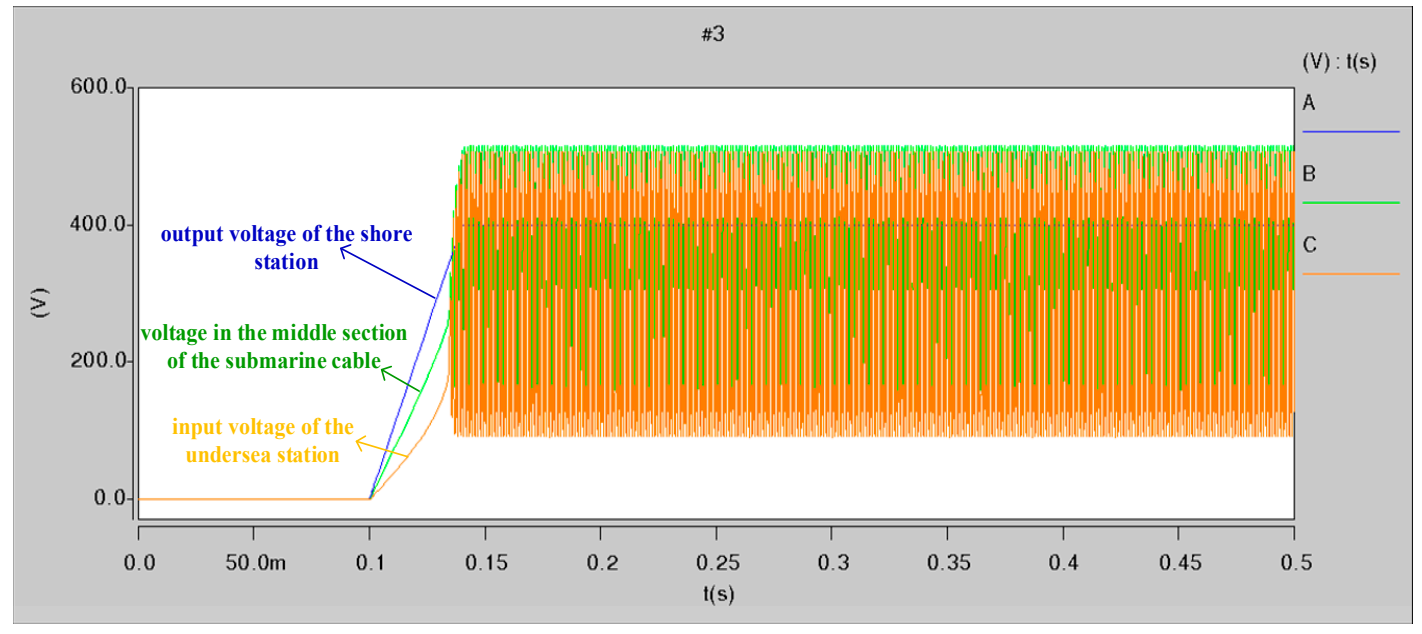

(e)

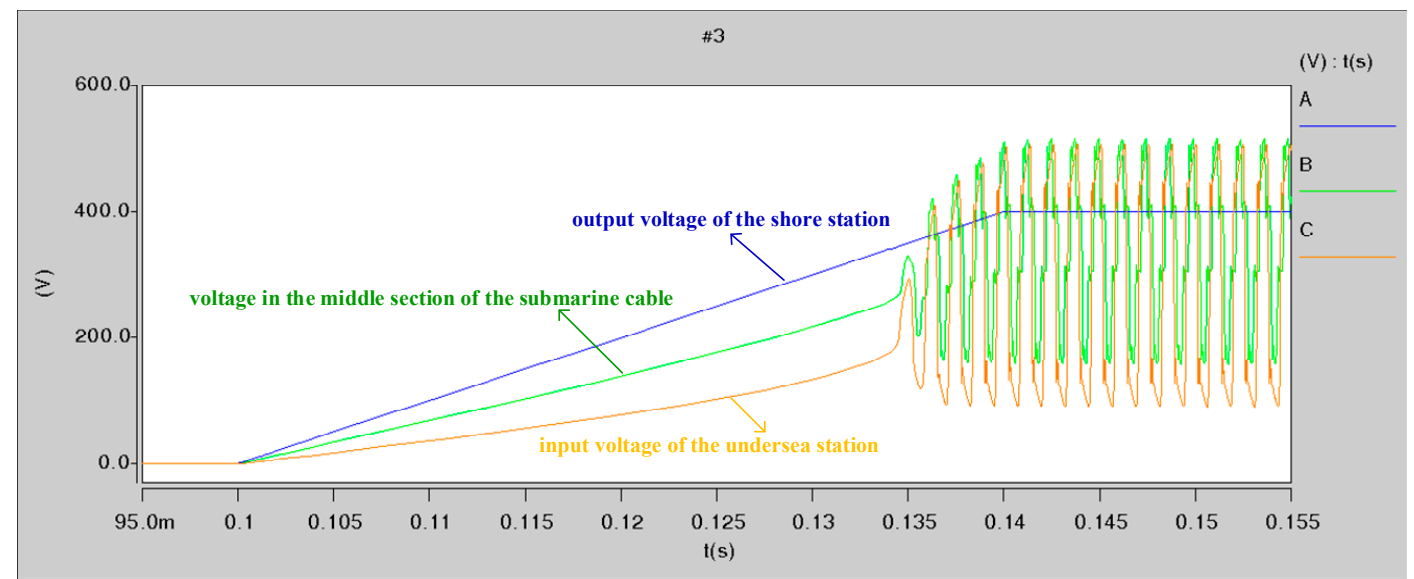

(f)

Figure 8. Cont. 


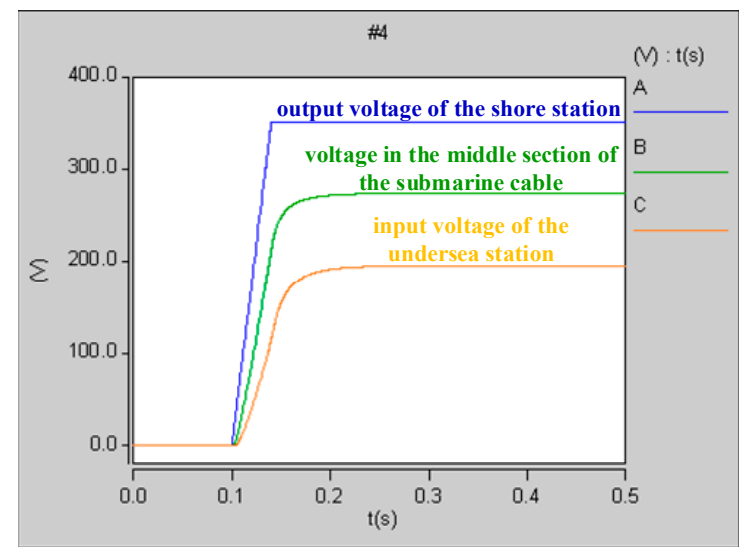

(g)

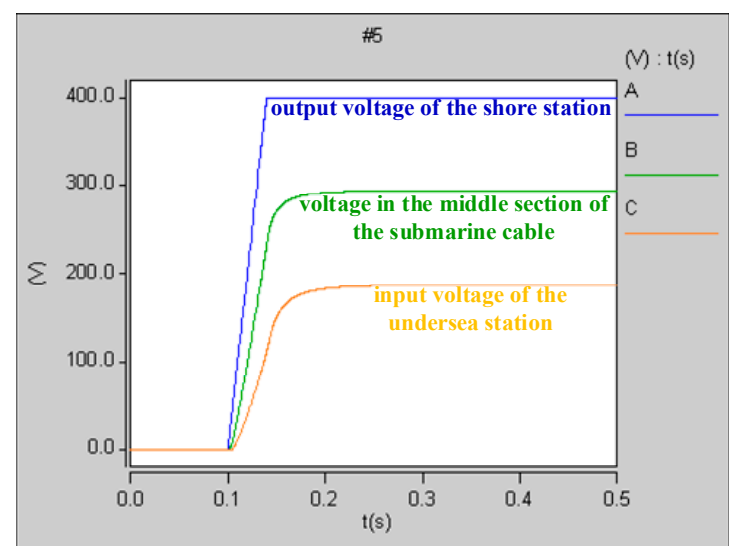

(h)

Figure 8. (a) The voltage transient processes in the parameter set \#1; (b) the current transient processes in the parameter set \#1; (c) the voltage transient processes in the parameter set \#2; (d) the current transient processes in the parameter set \#2; (e) the voltage transient processes in the parameter set \#3; (f) the magnified view of voltage transient processes in the parameter set \#3; (g) the voltage transient processes in the parameter set \#4; (h) the voltage transient processes in the parameter set \#5.

In Figure 8, the parameter set \#1 satisfies the criterion of the inequality Equation (29), so that its power system is stable in the case of power-on. The parameter sets \#2, \#3, \#4 and \#5 do not satisfy the criterion of the inequality Equation (29), so that their power systems are unstable in the case of power-on. It proves that the criterion of the inequality Equation (29) is valid. Moreover, the simulation results also show that the large-signal stability of a single-node CSO undersea DC power system is positively correlated with the output voltage of the shore station PFE and the capacitance of per unit length submarine cable, and negatively correlated with the power consumption of the undersea station and the inductance of per unit length submarine cable.

\subsection{Large-Signal Stability Experiments of a Single-Node CSO Undersea DC Power System}

An equivalent experimental prototype of a single-node CSO undersea DC power system was designed to verify the results of the above theoretical analysis and computer simulation. The equivalent experimental prototype is a medium-voltage simplified system model of a single-node CSO undersea DC power system, as shown in Figure 6. Its experimental configuration block diagram is shown in Figure 9. The shore station PFE is represented by an AC $220 \mathrm{~V}$ to DC $400 \mathrm{~V}$ converter. Every cable segment of $10 \mathrm{~km}$ is represented by a $\pi$-type lumped parameter equivalent circuit, and 36 circuits are cascaded to represent the $360 \mathrm{~km}$ submarine cable. The undersea station is represented by a DC $240 \mathrm{~V}$ to DC $48 \mathrm{~V}$ converter, feeding power to an electronic load which represents the undersea science payloads.

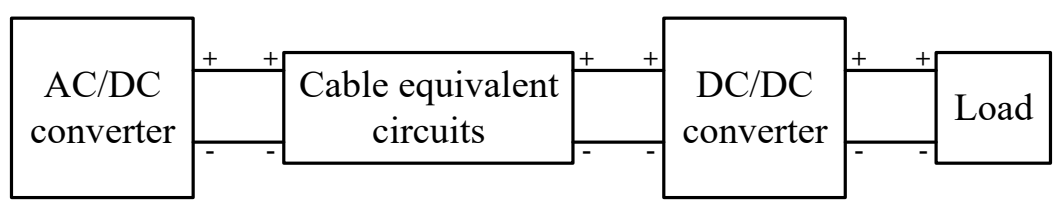

Figure 9. The block diagram of the prototype experiment configuration.

Five parameter sets for prototype experiments are applied to verify the effects of the shore station PFE output voltage, the undersea station power consumption and the submarine cable parameters on the large-signal stability of a single-node CSO undersea DC power system. The parameter sets are shown in Table 5. $U_{S}$ represents the output voltage of the AC/DC converter. $R_{0}, L_{0}$ and $C_{0}$ represent the resistance, inductance and capacitance of a cable segment respectively. $P_{L}$ represents the power consumption of the DC/DC converter with the electronic load. 
Table 5. Five parameter sets for prototype experiments.

\begin{tabular}{cccccc}
\hline No. & $\boldsymbol{U}_{\boldsymbol{S}} / \mathbf{V}$ & $\boldsymbol{P}_{\boldsymbol{L}} / \mathbf{W}$ & $\boldsymbol{R}_{\mathbf{0}} / \boldsymbol{\Omega}$ & $\boldsymbol{L}_{\mathbf{0}} / \mathbf{m H}$ & $\boldsymbol{C}_{\mathbf{0}} / \boldsymbol{\mu F}$ \\
\hline$\# 1$ & 400 & 82.29 & 10.2 & 4 & 2.03 \\
$\# 2$ & 400 & 82.29 & 10.5 & 400 & 2.03 \\
$\# 3$ & 400 & 82.29 & 10.2 & 4 & 0.016 \\
$\# 4$ & 350 & 82.29 & 10.2 & 4 & 2.03 \\
$\# 5$ & 400 & 96 & 10.2 & 4 & 2.03 \\
\hline
\end{tabular}

In the experiments, we assumed the startup of the AC/DC converter had encountered a large disturbance. The oscilloscope \#1 and \#2 were used to record the voltage and current transient processes in the prototype. The experiment results for the five sets are shown in Figure 10. In Figure 10, the red line indicates the output voltage of the AC/DC converter. The blue line indicates the voltage in the middle section of the submarine cable. The black line indicates the input voltage of the DC/DC converter. The green line indicates the output voltage of the DC/DC converter. The yellow line indicates the output current of the AC/DC converter.

Comparing the results of the five parameter sets for prototype experiments, it can be seen that during the startup of the AC/DC converter, the parameter sets \#1 and \#2 were stable. The parameter set \#3 would return to the steady-state equilibrium point after a period of voltage oscillation. According to the curve of the input voltage of the DC/DC converter, its oscillation amplitude is $8 \%$ of the steady-state voltage. But the parameter sets \#4 and \#5 would experience large fluctuations and were unable to return to a steady-state equilibrium point. According to the curve of the input voltage of the DC/DC converter, their oscillation amplitude is $15 \%$ of the steady-state voltage.

This leads to the conclusion that increasing the output voltage of the shore station PFE or the capacitance of the submarine cable, or decreasing the power consumption of the undersea station or the inductance of the submarine cable are beneficial to enhance the large-signal stability of a single-node CSO undersea DC power system.

\subsection{Large-Signal Stability Simulations of a Multi-Node CSO Undersea DC Power System}

In the last two sections, the large-signal stability of a single-node CSO undersea DC power system and the factors affecting the large-signal stability are studied. This section mainly studies the large-signal stability of a multi-node CSO undersea DC power system and its influencing factors.

In this paper, the large-signal stability of a multi-node CSO undersea DC power system is simulated by using Saber. The simulation file is shown in Figure 11. The shore station PFE is modeled by an ideal voltage source. The submarine cable is modeled by cascaded lumped parameter equivalent circuits. The undersea station is modeled by CPLs. In this system, there are four CPLs, in which the distance between the CPL4 and the source is the longest, so that CPL4 gets the minimum energy from the source. The total length of the submarine cable is assumed to be $490 \mathrm{~km}$. Every cable segment of 10 $\mathrm{km}$ is represented by a $\pi$-type lumped parameter equivalent circuit.

To study the effects of various factors on the large-signal stability of a multi-node CSO undersea DC power system, five simulation sets are needed. The parameter sets for the Saber simulation are shown in Table 6. $U_{S}$ represents the output voltage of the source, i.e., the shore station PFE. $R_{0}$, $L_{0}$ and $C_{0}$ represent the resistance, inductance and capacitance of a cable segment, respectively. $P_{L}$ represents the power consumption of the CPL4, i.e., the undersea station with external science payloads. The power consumption of the four CPLs is equal.

Initially, $U_{S}$ in the system was $0 \mathrm{~V}$. At $0.1 \mathrm{~s}$, according to experimental needs, the voltage rose from $0 \mathrm{~V}$ to $10 \mathrm{kV}$ or $6 \mathrm{kV}$, and the voltage transient processes were generated. 


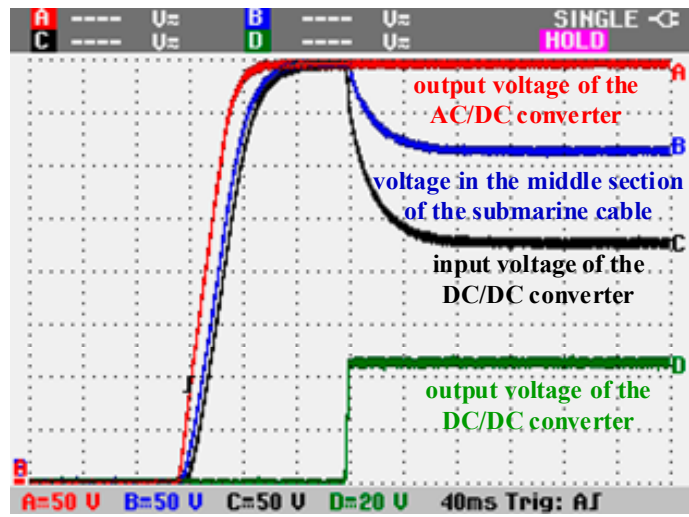

(a)

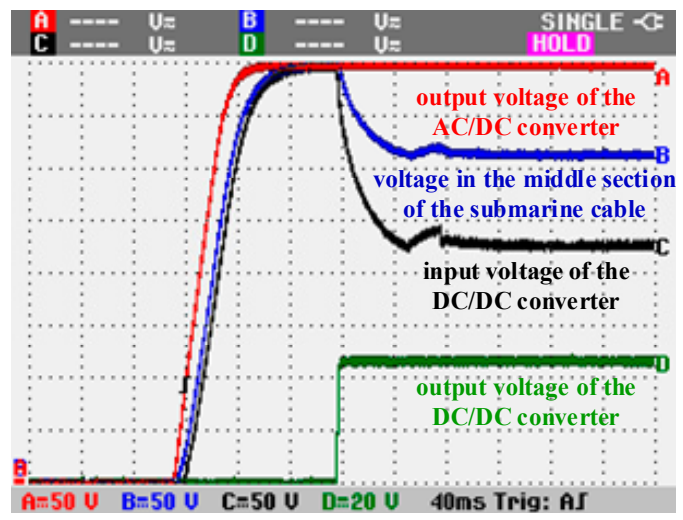

(c)

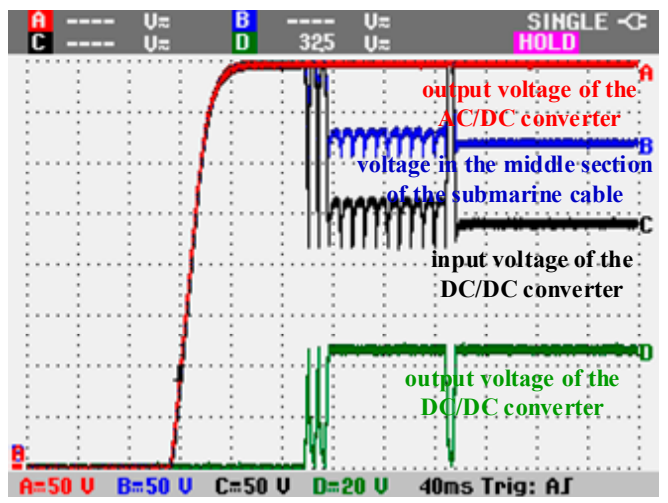

(e)

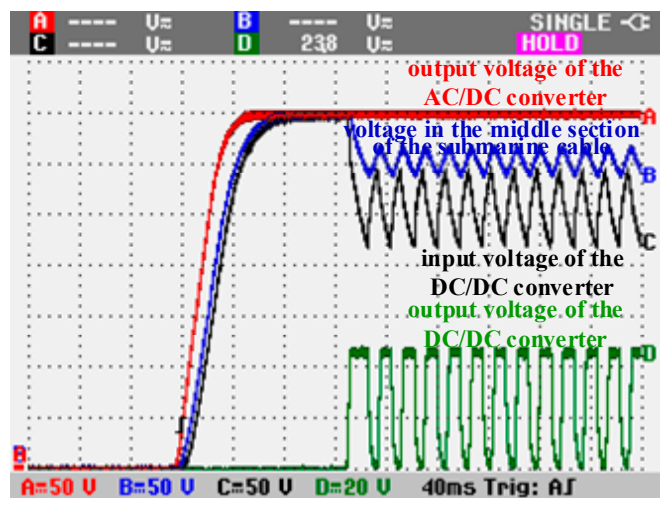

(g)

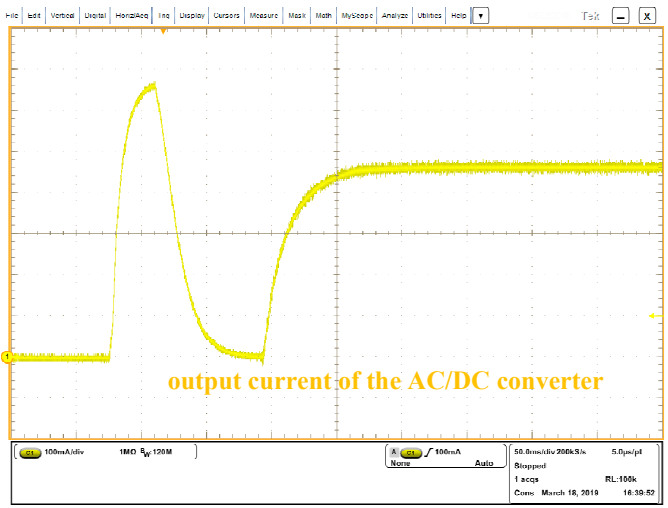

(b)

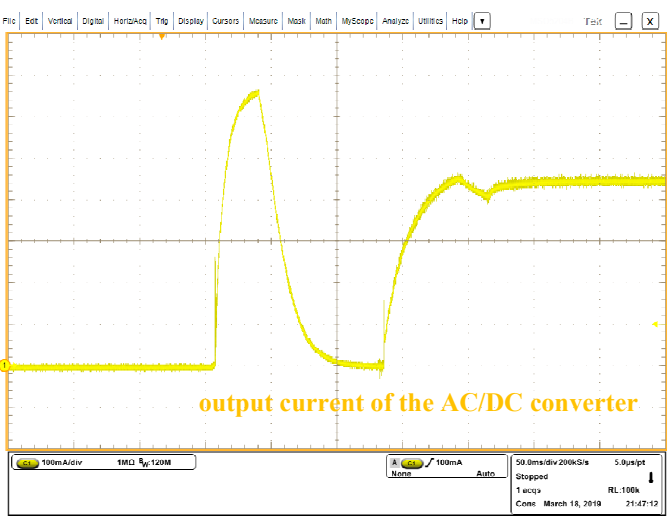

(d)

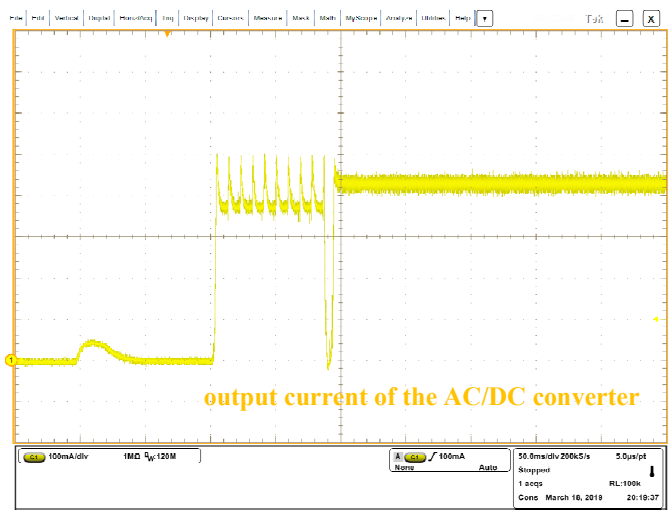

(f)

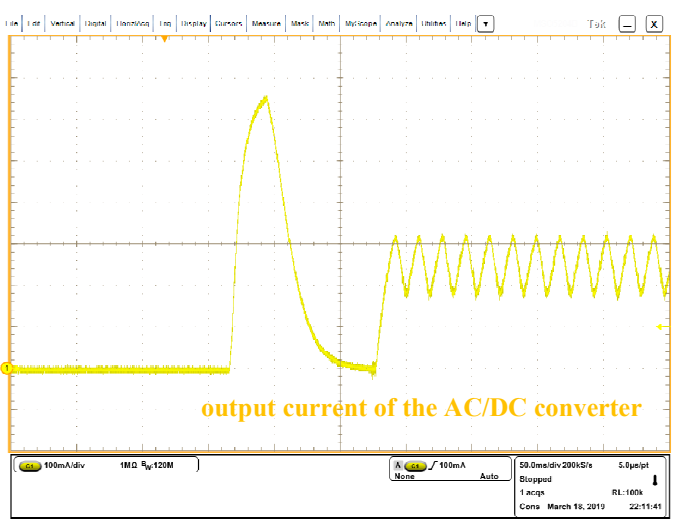

(h)

Figure 10. Cont. 


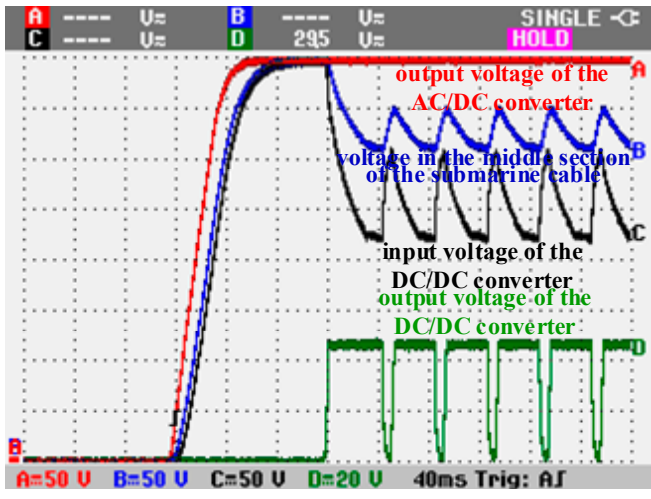

(i)

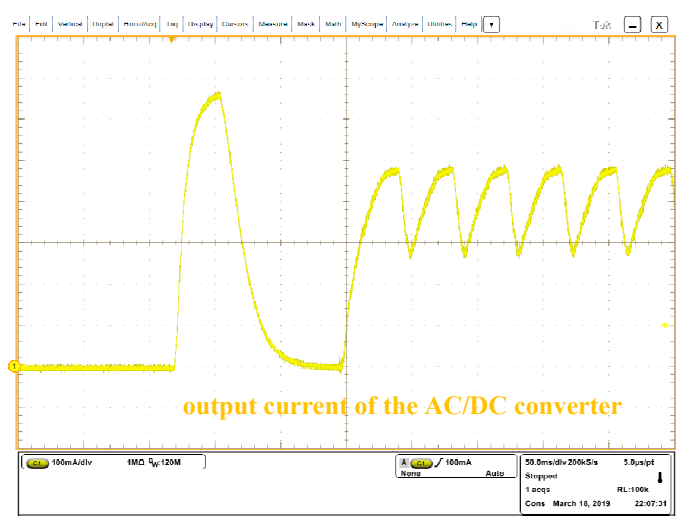

$(\mathbf{j})$

Figure 10. (a) The voltage transient processes in the parameter set \#1; (b) the current transient processes in the parameter set \#1; (c) the voltage transient processes in the parameter set \#2; (d) the current transient processes in the parameter set \#2; (e) the voltage transient processes in the parameter set \#3; (f) the current transient processes in the parameter set \#3; (g) the voltage transient processes in the parameter set \#4; (h) the current transient processes in the parameter set \#4; (i) the voltage transient processes in the parameter set \#5; (j) the current transient processes in the parameter set \#5.

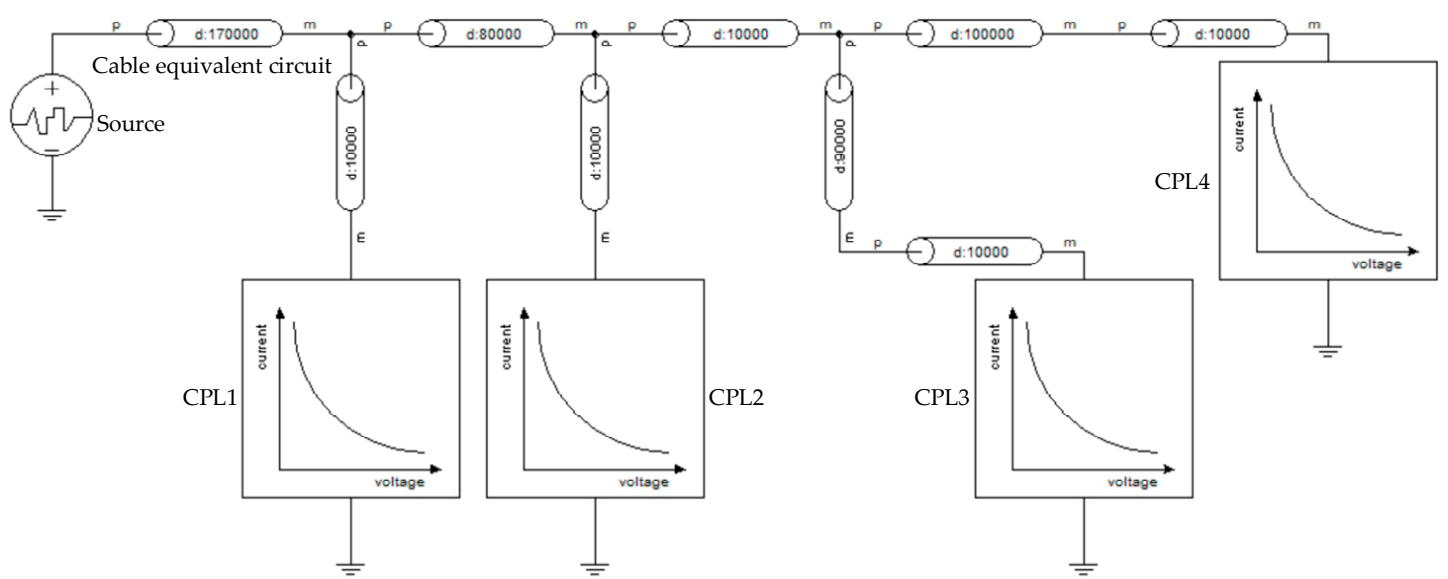

Figure 11. The Saber file for the large-signal stability simulation of a multi-node CSO undersea DC power system.

The simulation results are shown in Figure 12. It shows the voltage transient process of CPL4, where the green, purple, blue, orange and black lines represent the simulation results for the parameter

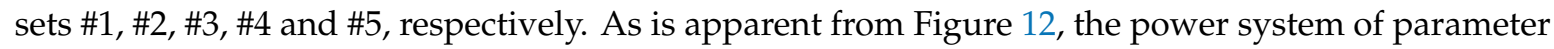
set \#1 was stable and the power systems of parameter sets \#2, \#3, \#4 and \#5 were unstable, in the case of power-on. This indicates that the large-signal stability of a multi-node undersea DC power system is positively correlated with the output voltage of the shore station PFE and the capacitance of per unit length submarine cable, and negatively correlated with the power consumption of the undersea station and the inductance of per unit length submarine cable. This is consistent with the large-signal stability conclusion of a single-node undersea DC power system. 
Table 6. Five Saber parameter sets for large-signal stability simulation of a multi-node CSO undersea DC power system.

\begin{tabular}{cccccc}
\hline No. & $\boldsymbol{U}_{\boldsymbol{S}} / \mathbf{k V}$ & $\boldsymbol{P}_{\mathbf{L}} / \mathbf{k W}$ & $\boldsymbol{R}_{\mathbf{0}} / \boldsymbol{\Omega}$ & $\boldsymbol{L}_{\mathbf{0}} / \mathbf{m H}$ & $\boldsymbol{C}_{\mathbf{0}} / \boldsymbol{\mu F}$ \\
\hline$\# 1$ & 10 & 10 & 10 & 4 & 2 \\
$\# 2$ & 10 & 10 & 10 & 4000 & 2 \\
$\# 3$ & 10 & 10 & 10 & 4 & 0.001 \\
$\# 4$ & 6 & 10 & 10 & 4 & 2 \\
$\# 5$ & 10 & 50 & 10 & 4 & 2 \\
\hline
\end{tabular}

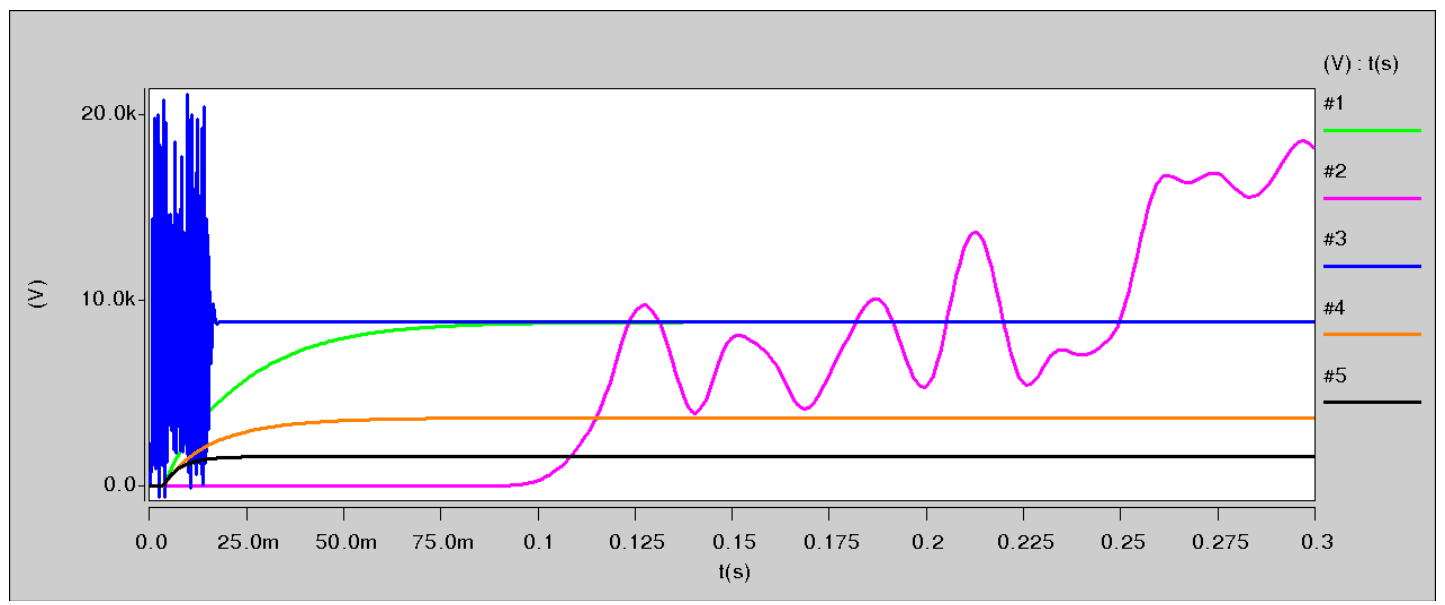

Figure 12. The voltage transient processes in five parameter sets.

\section{Conclusions}

By theoretical analysis and computer simulation, the equivalent physical model of a long-distance submarine cable is built. On this basis, a simplified model of a single-node CSO undersea DC power system is established. According to the simplified model, the steady-state equations of a single-node CSO undersea DC power system are derived. The steady-state equilibrium operation point criterion of the system is obtained, and has been verified in the medium-voltage experimental prototype of the single-node CSO undersea DC power system. Considering the characteristics of long-distance submarine cable, the Lyapunov function of the single-node CSO undersea DC power system is constructed by the mixed potential function method. The large-signal stability criterion of the system is derived according to the mixed potential function third theorem, and the validity of this criterion is verified by computer simulations and experiments. The factors affecting the large-signal stability are theoretically analyzed, and the conclusions are proved by computer simulations and prototype experiments: For a single-node CSO undersea DC power system, increasing the output voltage of the shore station PFE or the capacitance of the submarine cable is beneficial to enhancing the large-signal stability, and so is decreasing the power consumption of the undersea station or the inductance of the submarine cable. Furthermore, the simulation proves that the conclusion is also applicable to the large-signal stability of multi-node CSO undersea DC power systems.

Author Contributions: Conceptualization, F.L.; methodology, Y.J. and F.L.; software, Y.J.; validation, F.L.; formal analysis, Y.J.; investigation, Y.J. and F.L.; resources, F.L.; writing-original draft preparation, Y.J.; writing-review and editing, Y.J. and F.L.; visualization, Y.J.; supervision, F.L.; funding acquisition, F.L.

Funding: This research was funded by the Shanghai Science and Technology Commission for the fund of Shanghai science and technology innovation action plan, grant number 16DZ1205000.

Conflicts of Interest: The authors declare no conflict of interest. 


\section{Nomenclature}

DC

$\mathrm{CSO}$

PFE

MARS

OOI

VENUS

NEPTUNE

DONET

ESONET

MACHO

ECS

SCS

CNSSO

AC

BU

SIIM

SI

DPS

POL

CPL

i

$u$

$t$

$\mu_{1}$

$\mu_{2}$

M

$\lambda(M)$

$-R_{L}$

$R$

L

C direct current

cabled seafloor observatory

power feeding equipment

Monetary Accelerated Research System

Ocean Observatory Initiative

Victoria Experimental Network

Under the Sea

North-East Pacific Time-series

Underwater Networked Experiment

Dense Oceanfloor Network System for

Earthquakes and Tsunamis

Seafloor observation network for

earthquakes and tsunamis along

the Japan Trench

European Seas Observatory Network

Marine Cable Hosted Observatory

East China Sea

South China Sea

Chinese national scientific seafloor

observatory

alternating current

branching unit

scientific instrument interface module

science instrument

distributed power system

point of load

constant power load

current

voltage

time

minimum value

minimum value

matrix

the eigenvalue of the matrix $\mathrm{M}$

the dynamic negative impedance

of the CPL

resistance

inductance

capacitance

\section{G}

$\mathrm{R}_{1}$

$\mathrm{L}_{1}$

$U_{S}$

$R_{0}$

$L_{0}$

$\mathrm{C}_{0}$

$P_{L}$

$U_{L}$

$I_{L}$

$n$

$P(i, u)$

$-A(i)$

$B(u)$

$D(i, u)$

$P_{u}$

$P_{i}$

$A_{i i}(i)$

$B_{u u}(u)$

$L_{n}$

$C_{n}$ conductance

the resistance of $1 \mathrm{~km}$ submarine cable the inductance of $1 \mathrm{~km}$ submarine cable the capacitance of $1 \mathrm{~km}$ submarine cable the characteristic frequency of the undersea DC power system the angular velocity of the undersea DC power system

the overall length of the submarine cable

the wave impedance

the propagation coefficient

the impedance of per unit length submarine cable

the admittance of per unit length submarine cable the impedance of a submarine cable segment the admittance of a submarine cable segment

$U_{S}$ represents the output voltage of the shore station.

the resistance of a cable segment

the inductance of a cable segment the capacitance of a cable segment the power consumption of the undersea station

the input voltage of the undersea station the input current of the undersea station the number of submarine cable segments the mixed potential function the current potential function of some non-energy storage elements in the circuit the voltage potential function of non-energy storage elements in the circuit the energy of capacitors and part of non-energy storage elements in the circuit partial derivative of $\mathrm{P}$ to $\mathrm{u}$ partial derivative of $\mathrm{P}$ to $\mathrm{i}$ the second derivative test of $\mathrm{A}(\mathrm{i})$ to $\mathrm{i}$ the second derivative test of $\mathrm{B}(\mathrm{u})$ to $\mathrm{u}$ diagonal arrays of branch inductance diagonal arrays of branch capacitance 


\section{References}

1. Massion, G.; Raybould, K. Mars: The monterey accelerated research system-gene massion and keith raybould (monterey bay aquarium research institute) explore a cabled ocean observing system for a new generation of ocean. Sea Technol. 2006, 47, 39-42.

2. Cowles, T.; Delaney, J.; Orcutt, J.; Weller, R. The ocean observatories initiative: Sustained ocean observing across a range of spatial scales. Mar. Technol. Soc. J. 2010, 44, 54-64. [CrossRef]

3. Howe, B.M.; Duennebier, F.K.; Lukas, R. The ALOHA cabled observatory. In Seafloor observatories: A New Vision of the Earth from the Abyss, 1st ed.; Favali, P., Beranzoli, L., Santis, A., Eds.; Springer: Berlin, Germany, 2015; pp. 439-464.

4. Dewey, R.; Round, A.; Macoun, P.; Vervynck, J.; Tunnicliffe, V. The VENUS cabled observatory: Engineering meets science on the seafloor. In Proceedings of the MTS/IEEE Ocean, Vancouver, BC, Canada, 29 September-4 October 2007.

5. Barnes, C.R.; Best, M.M.; Johnson, F.R.; Pautet, L.; Pirenne, B. Challenges, benefits, and opportunities in installing and operating cabled ocean observatories: Perspectives from NEPTUNE Canada. IEEE J. Ocean. Eng. 2013, 38, 144-157. [CrossRef]

6. Kaneda, Y. The advanced ocean floor real time monitoring system for mega thrust earthquakes and tsunamis-application of DONET and DONET2 data to seismological research and disaster mitigation. In Proceedings of the Mts/IEEE Ocean, Seattle, WA, USA, 20-23 September 2010.

7. Kanazawa, T. The Japan trench earthquake and tsunami monitoring network of cable-linked 150 seafloor observatories (the S-net Project). Sci. Technol. 2015. [CrossRef]

8. Person, R.; Favali, P.; Ruhl, H.; Beranzoli, L.; Rolin, J.F.; Waldmann, C.; Huber, R.; Auffret, Y.; Çağatay, M.N.; Cannat, M. From ESONET multidisciplinary scientific community to EMSO novel European research infrastructure for ocean observation. In Seafloor Observatories: A New Vision of the Earth from the Abyss, 1st ed.; Favali, P., Beranzoli, L., Santis, A., Eds.; Springer: Berlin, Germany, 2015; pp. 531-563.

9. Zhang, Y.W.; Fan, D.D.; Xu, H.P. Records of the tsunami induced by the 2010 Chilean earthquake from Xiaoqushan seafloor observatory in the East China Sea. Chin. Sci. Bull. 2011, 56, 2957-2965. [CrossRef]

10. Hsu, S.K.; Lee, C.S.; Shin, T.C.; Liu, C.S.; Huang, B.S.; Kuo, B.Y.; Lin, C.H.; Tang, D.; Chang, H.Y.; Kuo, C.H. Marine cable hosted observatory (MACHO) project in Taiwan. In Proceedings of the Symposium on Underwater Technology and Workshop on Scientific Use of Submarine Cables and Related Technologies, Tokyo, Japan, 17-20 April 2007.

11. Lyu, F.; Peng, X.T.; Zhou, H.Y.; Yue, J.G.; He, B. Design of a prototype system for cabled seafloor observatory networks. Chin. J. Sci. Instrum. 2012, 33, 1134-1140.

12. Lu, S.H. Infrastructure, Operations, and Circuits Design of an Undersea DC Power System. Ph.D. Thesis, University of Washington, Washington, WA, USA, 2006.

13. Marx, D.; Magne, P.; Nahid-Mobarakeh, B.; Pierfederici, S.; Davat, B. Large signal stability analysis tools in DC power systems with constant power loads and variable power loads: A review. IEEE Trans. Power Electron. 2012, 27, 1773-1787. [CrossRef]

14. Magne, P.; Marx, D.; Nahid-Mobarakeh, B.; Pierfederici, S. Large-signal stabilization of a DC-link supplying a constant power load using a virtual capacitor: Impact on the domain of attraction. IEEE Trans. Ind. Appl. 2012, 48, 878-887. [CrossRef]

15. Griffo, A.; Wang, J.B. Large signal stability analysis of more electric aircraft power systems with constant power loads. IEEE Trans. Aerosp. Electron. Syst. 2012, 48, 477-489. [CrossRef]

16. Gholdston, E.W.; Karimi, K.; Lee, F.C.; Rajagopalan, J.; Panov, Y.; Manners, B. Stability of large DC power systems using switching converters, with application to the international space station. In Proceedings of the Energy Conversion Engineering Conference, Proceedings of the 31st Intersociety, Washington, WA, USA, 11-16 August 1996.

17. Ciezki, J.G.; Ashton, R.W. Selection and stability issues associated with a navy shipboard DC zonal electric distribution system. IEEE Trans. Power Deliv. 2000, 15, 665-669. [CrossRef]

18. Ahmed, S.; Kim, H.; Prohaska, T.; Ronkainen, T. Stability study of electric vehicle power electronics based power system. In Proceedings of the Electric Vehicle Conference (IEVC), Santa Clara, CA, USA, 23-25 October 2013. 
19. Liu, F.; Xiong, L.; Deng, L.H.; Feng, Y.; Wu, X.P. Vehicle stability criterion based on phase plane method. J. South China Univ. Technol. 2014, 42, 63-70.

20. Kabalan, M.; Singh, P.; Niebur, D. Large signal Lyapunov-based stability studies in microgrids: A review. IEEE Trans. Smart Grid 2017, 8, 2287-2295. [CrossRef]

21. Brayton, R.K.; Moser, J.K. A theory of nonlinear networks. I. Q. Appl. Math. 1964, 22, 1-33. [CrossRef]

22. Weiss, L.; Mathis, W.; Trajkovic, L. A generalization of Brayton-Moser's mixed potential function. IEEE Trans. Circuits Syst. I: Fundam. Theory Appl. 1998, 45, 423-427. [CrossRef]

23. Blanco, Y.; Perruquetti, W.; Borne, P. Stability and stabilization of nonlinear systems and Takagi-Sugeno's fuzzy models. Math. Probl. Eng. 2007, 7, 221-240. [CrossRef]

24. Loop, B.P.; Sudhoff, S.D.; Zak, S.H.; Zivi, E.L. Estimating regions of asymptotic stability of power electronics systems using genetic algorithms. IEEE Trans. Control Syst. Technol. 2010, 18, 1011-1022. [CrossRef]

25. Bacha, A.; Jerbi, H.; Braiek, N.B. An approach of asymptotic stability domain estimation of discrete polynomial systems. In Proceedings of the Computational Engineering in Systems Applications, Beijing, China, 4-6 October 2006.

26. Zhang, K.D. Research on the Distributed Parameter Circuit Model and its Application in UHV Transmission Line Protection. Master's Thesis, Chongqing University, Chongqing, China, 2014.

27. Chan, T. Analytical Methods for Power Monitoring and Control in an Underwater Observatory. Ph.D. Thesis, University of Washington, Washington, WA, USA, 2007.

28. Jeltsema, D.; Scherpen, J.M.A. On Brayton and Moser's missing stability theorem. IEEE Trans. Circuits Syst. Express Briefs 2005, 52, 550-552. [CrossRef]

29. Liu, X.B.; Zhou, Y.J.; Zhang, W. Large signal stability criteria for constant power loads with damped filters. Trans. China Electrotech. Soc. 2011, 26, 155-161.

30. Liu, X.B.; Zhou, Y.J.; Zhang, W.; Ma, S.H. Stability criteria for constant power loads with multistage LC filters. IEEE Trans. Veh. Technol. 2011, 60, 2042-2049. [CrossRef]

(C) 2019 by the authors. Licensee MDPI, Basel, Switzerland. This article is an open access article distributed under the terms and conditions of the Creative Commons Attribution (CC BY) license (http://creativecommons.org/licenses/by/4.0/). 